\title{
Research on Multi-level Domain Name Comprehensive Information Management
}

\author{
Chunqiang $\mathrm{Li}$ \\ School of Information Management, Beijing Information Science and Technology University, Beijing, \\ 100101, China \\ 642510571@qq.com
}

Keywords: multi-level; domain name system; information management; domain name sturcture; system function; logical structure

\begin{abstract}
Domain name system are both the underlying business, but also an important part of the Internet infrastructure. Research for this article expand BIND domain name system management problems. First, four domain names, for example, study the structure of multi-level domain name system; Then, based on the logical structure of the database Oracle database system, design information management domain; Finally, the design of the functional structure of the domain name system management, user management, information maintenance and data maintenance subsystem configuration. The study of the application domain to achieve, delete, review, approval, change, cancellation, opened, disable functions such as integrated management, improve management efficiency and level of information, to provide solutions for specifications, safety and integrated management of domain names.
\end{abstract}

\section{Introduction}

Domain name, is the name of the Internet consists of a string of names separated by dots composed of a computer or group of computers for electronic data transmission azimuth identify computers, sometimes also referred to as location, geographical name or administrative autonomy a local area right. The purpose of the establishment of the domain name is the address easy to remember and to communicate a group of servers. DNS (Domain Name System) is both a basic business of the Internet, but also an important part of the Internet infrastructure, complete the mapping host names and Internet network addresses. Almost all of the interconnection software are in use DNS, such as e-mail, remote terminal program, file transfer programs and Web browsers.

Bind (Berkeley Internet Name Domain) is an open-source DNS server software by the University of California, Berkeley, developed and maintained, is currently the most widely used DNS server software in the world, supports a variety of Unix and Windows platforms. BIND DNS protocol specification as an example of realization, feature-rich, powerful, safe and strong. But there are also inadequate BIND system, domain name, domain name record data, adopted a text file storage, maintenance of the domain name is the text file management; Operation and maintenance of domain names in manual operation, maintainability, error-prone, inefficient, difficult to manage and maintain; Operation and maintenance costs are high, and the management of professional skills in BIND has a certain requirement; Domain name system itself is not the domain name records with immediate effect and the management mechanism and function, domain name every change to manual processing. Lack of data security protection mechanism, no domain name data backup and restore function; No domain name registration, authentication function, can not achieve the domain name registration and filing [1,2]. In view of the problems existing in the management of the domain name to construct multi-level domain information management system, for the specification of the domain name, the security and the integrated management to provide solutions.

\section{System Structure on Multi-level Domain Name System}

Two correspondence between IP address and domain name: "One to one" relationship, IP Internet address is unique, an IP address corresponds to the only one host. Given a domain name address can 
find a unique IP address; "One to many" relationship, a computer to provide more services, both as a server and as a mail server www. IP address, or unique, but it can be given a different name according to multiple services in a computer, an IP address corresponding to multiple domain names. DNS is the domain name space to the site IP, so that people can easily access to the website by registering the domain name of a service. IP address is a numeric address on the network to identify sites, in order to facilitate memory, the use of the domain name instead of the IP address identifies the site address. Domain name resolution is the domain name to the IP address of the conversion process. The resolution of the domain name is completed by the DNS server.

The domain name is easy to remember and understand, make the network service easier to manage in the application and the IP address of the equivalent. The domain name uses the hierarchical naming mechanism, the name space is divided into several parts, and authorized the corresponding organization management. The syntax of the domain name system is "the host name. The level n sub domain name... Second sub domain name domain name." The authority has the right to further division of the jurisdiction of the name space, and then the corresponding management authority. Domain name system is configured for a tree based distributed database, the leaf node is host name, the intermediate node is the appropriate level of domain name server, the root node is empty. Domain name use (.) segmentation. Multi-level domain name system structure as shown in Fig. 1 [3-6].

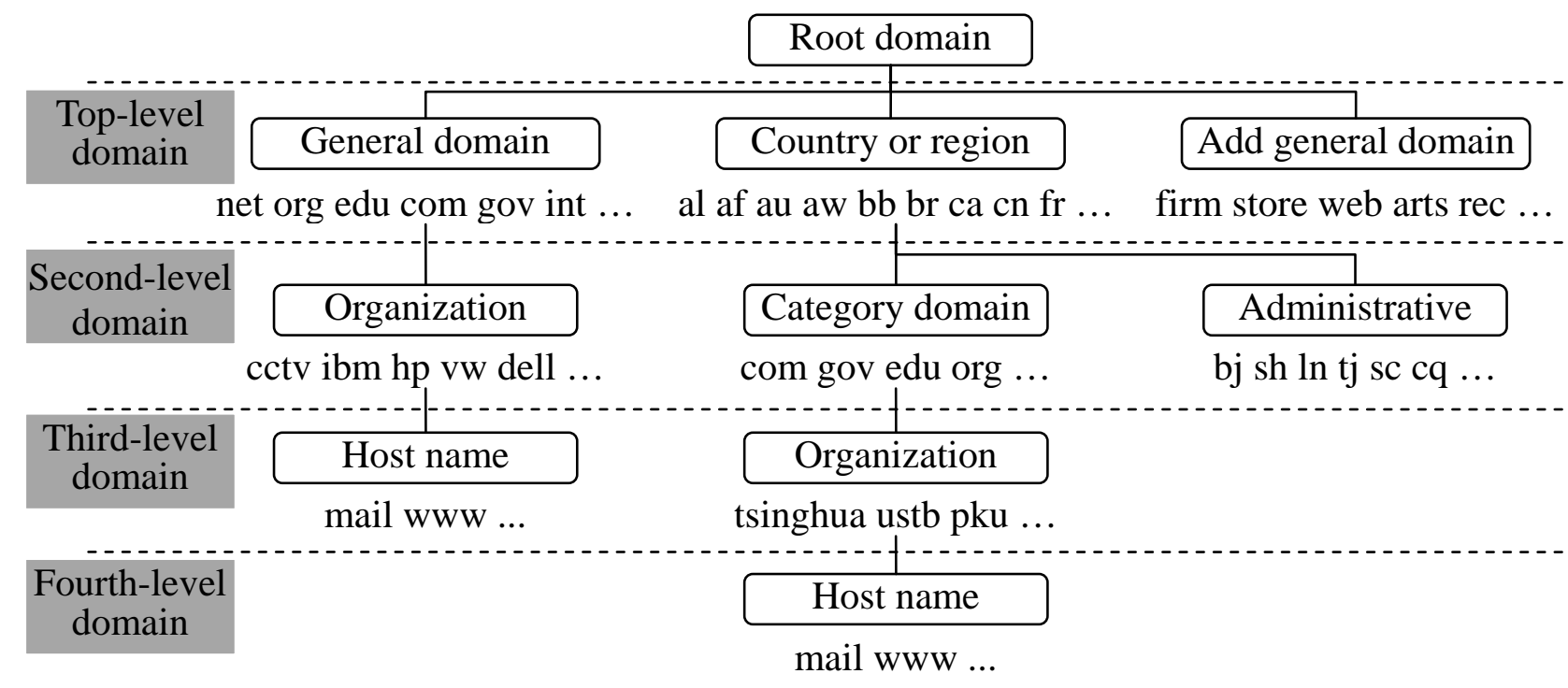

Fig. 1. System structure on multi-level domain name system

The top-level domain is a standardized symbol to ensure the generality of the domain name system. Top-level domain is divided into three categories [7,8]: Is a generic top-level domain, for example, says the business enterprise com, said network provider net, said the nonprofit organization org; Second, the country code Top Level Domain, over 200 countries or regions are allocated in accordance with ISO3166 country code top-level domain. For example, China is cn, the United States is us, Japan is jp, tw Taiwan is like; Third, the new generic top-level domains, for example, indicate info information services, said the company and the corporate firm, represents a personal nom, represents the arts and other cultural and entertainment.

Secondary domain name is refers to under the top-level domain name domain name, in generic top-level domain name, refers to the online name of the domain name registrant, such as cctv, ibm, yahoo, and microsoft. Most of the domain name dispute occurred in the com top-level domain, because most Internet companies for the purpose of profit. In countries or regions of the top-level domain is divided into two categories, one category is the domain name, and the meaning is the same as the same generic top-level domain; Second, the administrative domain, such as Beijing is bj, Shanghai is sh, Tianjin is tj.

Third-level domain with the letter (A - Z, a - z, capitalization, etc.), numbers (0 to 9) and hyphen (-) which are connected by a solid point between all levels of domain, domain III (.) not more than 
20 characters in length. If there is no special reason, we recommend using the applicant's English name (or initials) or pinyin name (or initials) as a third-level domains, in order to maintain the domain name of clarity and simplicity.

Register domain names need to follow the principle of first come, first registration, domain name is a valuable resource, in the new economic environment, business sense domain name has been far greater than the technical sense, and become involved in international science and technology under the new conditions important means of market competition. At the same time, the domain name is an intellectual achievement, there is a literal meaning commercial marks, and trademarks, similar names, it reflects the considerable creativity. From a global perspective, even though national legislation has not yet put the domain name as the exclusive rights to be protected, but the international domain name system is to develop coordination through the World Intellectual Property Organization, which fully shows that people have the domain name as part of intellectual property.

\section{Design on database logical structure}

Typically the data structure design includes conceptual design, logical design, physical design and design security and confidentiality. Logical structure design task is to design the conceptual structure of the result is converted to and selection of database management systems supported by the data model consistent logical structure. Relational model and relational database management systems have been widely accepted and widely used. The system uses the Oracle relational database management system. Oracle is the database has been a leader in the field of products, is the world's most popular relational database management systems, portability, ease of use, functionality, suitable for all types of large, medium and small, the microcomputer environment. At the same time, but also a high efficiency, good reliability, adapt to high throughput database solutions. Logical structure of the system design results shown in Table 1. 
Table 1. Database logical structure on multi-level domain name

\begin{tabular}{|c|c|c|c|c|c|c|c|}
\hline No & Field name & Data type & Byte & No & Field name & Data type & Byte \\
\hline \multicolumn{4}{|c|}{ Basic information } & \multicolumn{4}{|c|}{ Administrator information } \\
\hline 1 & Sate & varchar2 & 10 & 41 & AdministratorName & varchar2 & 20 \\
\hline 2 & ApplyClass & varchar2 & 10 & 42 & AdministratorUnit & varchar2 & 50 \\
\hline 3 & SystemName & varchar2 & 50 & 43 & AdministratorMailing & varchar2 & 50 \\
\hline 4 & DomainClass & varchar2 & 10 & 44 & AdministratorPostcode & varchar2 & 6 \\
\hline 5 & ApplyGrade & varchar2 & 10 & 45 & AdministratorPhone & varchar2 & 50 \\
\hline 6 & SecondLevelDomain & varchar2 & 50 & 46 & AdministratorFax & varchar2 & 50 \\
\hline 7 & ThirdLevelDomain & varchar2 & 50 & 47 & AdministratorEmail & & 50 \\
\hline 8 & FourthLevelDomain & varchar2 & 50 & \multicolumn{4}{|c|}{ Applicant information } \\
\hline 9 & HostName & varchar2 & 50 & 51 & ApplicantName & varchar2 & 20 \\
\hline 10 & SubCenterId & number & 4,0 & 52 & ApplicantUnitName & varchar2 & 50 \\
\hline 11 & TransmissionState & varchar2 & 10 & 53 & ApplicantMailing & varchar2 & 50 \\
\hline \multicolumn{4}{|c|}{ Change and IP address information } & 54 & ApplicantPostcode & varchar2 & 6 \\
\hline 21 & BeforeDomainName & varchar2 & 50 & 55 & ApplicantPhone & varchar2 & 50 \\
\hline 22 & AfterDomainName & varchar2 & 50 & 56 & ApplicantFax & varchar2 & 50 \\
\hline 23 & IPAddress & varchar2 & 15 & 57 & ApplicantfEmail & varchar2 & 50 \\
\hline 24 & SpareIPAddress & varchar2 & 15 & 58 & ApplyDateTime & date & \\
\hline 25 & PortNumber & varchar2 & 10 & \multicolumn{4}{|c|}{ Approval information } \\
\hline \multicolumn{4}{|c|}{ Apply unit information } & 61 & ApprovalDateTime & date & \\
\hline 31 & ApplyPurpose & varchar2 & 100 & 62 & ApprovalPeople & varchar2 & 20 \\
\hline 32 & ApplyUnitName & varchar2 & 50 & 63 & ApprovalUnit & varchar2 & 50 \\
\hline 33 & ApplyUnitShort & varchar2 & 50 & \multicolumn{4}{|c|}{ Other information } \\
\hline 34 & ApplyUnitPerson & varchar2 & 50 & 71 & OrganizationID & number & 4,0 \\
\hline 35 & ApplyUnitMailing & varchar2 & 100 & 72 & ServiceAddress & varchar2 & 50 \\
\hline 36 & ApplyUnitPostcode & varchar2 & 6 & 73 & ServiceDescription & varchar2 & 50 \\
\hline 37 & ApplyUnitAddress & varchar2 & 100 & 74 & Remarks & varchar2 & 80 \\
\hline
\end{tabular}

As can be seen from Table 1, the logical structure of the system consists of seven categories of information, namely "Basic information, Change and IP address information, Apply unit information, Administrator information, Applicant information, Approval information, Other information", for each type of information By including the number of fields, such as multi-level domain name system information to provide a complete storage structure.

\section{Function on Multi-level Domain Name System}

Functional design commonly used method is simulated. Blocking is one of the most important design thought, the complex system is decomposed into a number of smaller, simpler, easier to establish and modify the part. On the one hand, each module has relative independence, which can be designed and implemented separately; On the other hand, the relationship between the modules is explained in a certain way. Each module in the constraints of these relationships together constitute a unified whole, complete the system functions. Structure and function is the simulation results, the system functions are decomposed, represented by the functional dependency graph, figure in each a rectangular frame is called a function module. Management information system of the system can be viewed as a function of the target system is lower, and the each function can also continue to decompose for more functions. Functional decomposition process from large to small, 
from coarse to fine, from top to bottom. Conceptually speaking, the upper layer functions to control the lower function, the more the upper layer function is more general, the lower the function more specific. Functional decomposition is a from the abstract to the concrete, from the simple to the complex process. Function module can according to the specific circumstances of large some or some small decomposition was the smallest functional module can be a program of each process, and big function module can complete a task is a set of procedures to be. The system function structure as shown in Fig. 2.

The core function of the system is the domain name management, mainly includes 8 functions $[9,10]$. Among them, the domain name delete for the applicant to fill in the relevant information, mainly is to fill in the "Database logical structure" in the "Basic information"; Name delete Domain, for the domain name in the absence of review or approval before the applicant to delete their own application information; Domain name check for domain name management audit domain name application information, the audit results including "by" and "through", if "through" the reasons shall be explained; Domain name approval for approval unit person in charge of the audit by domain name approved, the result is "approved" and "not approved", if "not approved" to explain why. Name change Domain, for the application to the management department to submit domain name change information; Domain name cancellation for management of domain name completely removed, the applicant if need to use, only to re apply for; Name enlightened Domain, so that the use of the domain name has been approved to enter the state can be used; Domain name disable, for violation of the provisions of the domain name, management department can stop using the use of units can also apply to stop using.

Auxiliary function of the system is Information maintenance, User management and Data maintenance. Wherein, Information maintenance, standardization and code is run-time information systems to add, modify and delete operations; User management, the basic information of the user entry permissions, and other information management, as well as the user to modify the password; Data maintenance, the maintenance of the system log and data, including database backup and recovery, configuration file backup and recovery. 


\begin{tabular}{|c|c|}
\hline Domain name disable & Organization index \\
\hline Domain name enlightened & Organization code \\
\hline Domain name cancellation & Approval unit \\
\hline Domain name change & Apply unit \\
\hline Domain name approval & Domain name level \\
\hline Domain name check & Domain name class \\
\hline Domain name delete & Apply grade \\
\hline Domain name apply & Apply class \\
\hline Domain name management & Information maintenance \\
\hline \multicolumn{2}{|c|}{ Multi-level domain name management system } \\
\hline User management & Data maintenance \\
\hline User login & Log management \\
\hline User modify password & Database backup \\
\hline User login information query & Database recovery \\
\hline User information management & Configuration file backup \\
\hline User privilege management & Configuration file recovery \\
\hline
\end{tabular}

Fig. 2. Function structure on multi-level domain name system

\section{Conclusion}

With the development of the Internet, a large number of enterprises by setting up their own website to promote their products and services. Domain name and enterprise, the product has a closer relationship, has become the enterprises to enter the information society, to adapt to the modern market competition important tool, its commercial value more and bigger. Domain name management has become the Internet field complex and important work, this paper aiming at the domain name open source domain name server bind deficiencies in the management function of the design domain information management system database logical structure and system function, domain application, delete, review, approval, modification, cancellation, open, disable function integration management and improve the management efficiency and the level of information technology, to avoid the conflict of domain name and repeated applications, has certain theoretical and practical significance.

\section{Acknowledgement}

This work is supported by Major special project of nuclear high-base (2012ZX01039-004-48); School fund of Beijing information science and technology university (0925020).

\section{References}

[1] Z. L. Guo, X. F. Li, "Regional domain name system construction and management," Fujian Computer, vol. 25, no. 1, pp. 43-44, 2009. 
[2] D. R. Zhou, L. Xia, T. Shu, et al, "Research and Design of Domain Name Management System Based on BIND," Journal of Yangtze University (Natural Science Edition), vol. 8, no. 7, pp. 54-57, 2011.

[3] Yakup Koç, Almerima Jamakovic, Bart Gijsen, "A global reference model of the domain name system," International Journal of Critical Infrastructure Protection, vol. 5, no. 3, pp. 108-117, 2012.

[4] Casey Deccio, "Maintenance, mishaps and mending in deployments of the domain name system security extensions (DNSSEC)," International Journal of Critical Infrastructure Protection, vol. 5, no. 2, pp. 98-103, 2012.

[5] Baidu library , "Domain name system and domain name system," http://wenku.baidu.com/link?url=51_Ne9JVBcJRxlHnyPtGiXQ49F_Da_fV6QH0-mydKgdijhy Q4QRL9UC45BFB-GRq6E15pwUFcpUsOYYk7zGC5Wv-ZbYjlVOTWHdKhteTCTe, 2015-12-21.

[6] Network marketing teaching website, "The structure and level of the domain name," http://www.wm23.com/resource/r02/domain_2002.htm, 2015-12-21.

[7] Baidu Encyclopedia, "Domain Name," http://baike.baidu.com/link?url=fWO_IgL7WR1V31jE9VhgoiXp5GMcjGzsfYrZ0vOp83W-_z 9IyGGq2R8rjxMyHT3FmB-dzw5yZ30-H2_iJWYuBq, 2015-12-21.

[8] Undrah B. Baasanjav, "Linguistic diversity on the internet: Arabic, Chinese and Cyrillic script top-level domain names," Telecommunications Policy, vol. 38, no. 11, pp. 961-969, 2014.

[9] LI Min, NI Wei-min, HE Mei, et al, "Design and implementation of DNS management system based on .Net," Journal of Xiamen University (Natural Science), vol. 46, no. 2, pp. 118-120, 2007.

[10] Z. L. Yang, "Research and design of Web domain name management system based on BIND," Computer CD Software and Applications, vol. 15, no. 17, pp. 239-241, 2012. 